\title{
Internationale Datenschutzkonferenz stellt Datenschutzanforderungen an das automatisierte und vernetzte Fahren
}

Auf Initiative der Bundesbeauftragten für den Datenschutz und die Informationsfreiheit, Andrea Voßhoff, hat die 39. Internationale Datenschutzkonferenz in Hongkong grundlegende Datenschutzanforderungen für die Mobilität der Zukunft beschlossen.

Das Recht der Fahrzeugnutzer auf den Schutz ihrer Daten ist eine der zentralen Forderungen von Datenschutzbehörden aus 78 Staaten. Auf Initiative der Bundesbeauftragten für den Datenschutz und die Informationsfreiheit, Andrea Voßhoff, verabschiedeten sie während der 39. Internationalen Datenschutzkonferenz vom 25. bis 29. September 2017 in Hongkong eine Entschließung zum Datenschutz beim automatisierten und vernetzten Fahren.

\section{Appell an Industrie und Politik}

In 16 Punkten wendet sich die Konferenz an Automobilhersteller und -Zulieferer, Gesetzgeber und Behörden sowie an die Anbieter fahrzeugbezogener Internetdienste. Die Datenschützer appellieren, das Recht der Fahrzeugnutzer auf Privatsphäre und den Schutz ihrer personenbezogenen Daten in jeder Phase der Entwicklung und Herstellung neuer Produkte und Dienste zu beachten. Basierend auf den von der Bundesdatenschutzbeauftragten im Juni 2017 veröffentlichten Empfehlungen für den Datenschutz in automatisierten und vernetzten Fahrzeugen beschreibt die Entschließung, wie das informationelle Selbstbestimmungsrecht der Fahrzeugnutzer geschützt werden sollte.

Die Bundesdatenschutzbeauftragte Andrea Voßhoff dazu: Das Auto ist Symbol von Freiheit und Unabhängigkeit. Die Digitalisierung des Straßenverkehrs könnte dies grundlegend verändern. In modernen Fahrzeugen sammeln bereits heute unzählige Sensoren Daten zum Fahrverhalten und den zurückgelegten Wegen. Daraus lassen sich detaillierte Persönlichkeitsprofile er- stellen. Fahrerinnen und Fahrer müssen daher jederzeit die volle Hoheit über die Verwendung personalisierbarer Fahrzeugdaten haben. Grundsätzlich sollten sie über jede Datenverwendung vollständig und transparent unterrichtet werden. Dafür sind datenschutzgerechte Technologien und Voreinstellungen notwendig.

Mit der Entschließung definiert und fordert die internationale Datenschutzkonferenz die Einhaltung gemeinsamer internationaler Standards.

\section{Hintergrund zur internationalen Datenschutzkonferenz:}

Die Internationale Datenschutzkonferenz ist ein 1979 gegründetes globales Forum der Datenschutzaufsichtsbehörden, in welchem mittlerweile 120 Mitglieder aus 78 Staaten gemeinsame Positionen zum Datenschutz und zum Schutz der Privatsphäre formulieren. Die diesjährige 39. Konferenz findet unter dem Motto Connecting West with East in Respecting Data Privacy fand in Hongkong statt. Ein Bericht über weitere Themen und Entschließungen der Konferenz wird zeitnah auf der Homepage der BfDI abrufbar sein.

Hier finden Sie den Text der Entschließung (engl. Original) zum Download:

39th International Conference of Data Protection and Privacy Commissioners Hong Kong - https://www.bfdi.bund.de/SharedDocs/Publikationen/EN/InternationalDS/2017_39thIDSK_HongKong_ResolutionOnDataProtectionAutomatedAndConnectedVehicles.html?nn=5217154 